\title{
Plasma miR-451 with echocardiography serves as a diagnostic reference for pulmonary hypertension
}

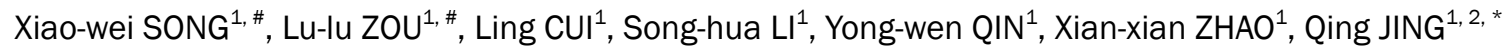 \\ ${ }^{1}$ Department of Cardiology, Changhai Hospital, Shanghai 200433, China; ${ }^{2}$ Key Laboratory of Stem Cell Biology, Institute of Health \\ Sciences, Shanghai Institutes for Biological Sciences \& Shanghai Jiao-Tong University School of Medicine, Chinese Academy of \\ Sciences, Shanghai 200031, China
}

\begin{abstract}
Due to the lack of typical clinical symptoms, the average delay time for diagnosis of pulmonary hypertension (PH) is longer than 2 years. It is urgent to find biomarkers for PH diagnosis. In this study we investigated whether plasma microRNAs (miRNAs) can be used as biomarkers for PH diagnosis. We used microarray to identify dynamic miRNAs between $\mathrm{PH}$ and non-PH patients. The candidate miRNAs were verified using qRT-PCR in a mouse model of PH, which was induced by monocrotaline (MCT) injection. We observed that miR-21, miR-126, miR-145, miR-191 and miR-150 had no differences between control mice and MCT-treated mice; but plasma miR-451 was significantly decreased in the 2 wk-MCT group, with no further decrease in the 4 wk-MCT group. Plasma miR-451 was also markedly decreased in PH patients, whereas miR-21, miR-126, miR-150 and miR-320 did not show differences between 53 PH patients and 54 non-PH patients. Receiver operating characteristic curves (ROCs) were constructed from the patient data to assess the clinical diagnostic values of circulating miR-451 and Doppler echocardiography (D-ECHO). The areas under the curve (AUCs) of ROCs for miR-451 and D-ECHO were 0.710 and 0.766 , respectively. Combination of miR-451 and D-ECHO with AUC of 0.825 was superior to the use of either miR-451 or D-ECHO alone for PH diagnosis. In conclusion, plasma miR-451 has a moderate diagnostic value in $\mathrm{PH}$ comparable to that of $\mathrm{D}-\mathrm{ECHO}$, and the combination of miR-451 with D-ECHO has better diagnostic value than either method alone, which may have implications for $\mathrm{PH}$ diagnosis.
\end{abstract}

Keywords: pulmonary hypertension; biomarker; miR-451; D-ECHO; miRNAs

Acta Pharmacologica Sinica (2018) 39: 1208-1216; doi: 10.1038/aps.2018.39; published online 24 May 2018

\section{Introduction}

Pulmonary hypertension $(\mathrm{PH})$ is characterized by progressive blood vessel remodeling and increasingly pulmonary vascular resistance $^{[1]}$. Long-standing $\mathrm{PH}$ leads to right heart failure or even sudden death. Long-term functional prognosis of $\mathrm{PH}$ is significantly better in patients with grade I or II heart dysfunction than that in patients with grade III or $\mathrm{IV}^{[2]}$, indicating that early diagnosis and intervention of $\mathrm{PH}$ are very important for improving $\mathrm{PH}$ therapeutic outcomes. However, the average delay time from symptoms to diagnosis of $\mathrm{PH}$ is longer than two years due to a lack of typical clinical symptoms and low morbidity ${ }^{[3]}$. Therefore, it is urgent to seek methods or biomarkers for early screening of high-risk patients with $\mathrm{PH}$.

Currently, the best-known method for the diagnosis of $\mathrm{PH}$ is right heart catheterization (RHC), with which pulmonary

\footnotetext{
"These authors contributed equally to this work.

*To whom correspondence should be addressed.

E-mail qjing@sibs.ac.cn

Received 2017-11-09 Accepted 2018-03-13
}

hemodynamic parameters can be directly obtained. It has been proposed that a mean pulmonary artery pressure (mPAP) greater than $25 \mathrm{mmHg}$ can serve as a standard for diagnosis of $\mathrm{PH}^{[4]}$. Though RHC is generally safe, it still poses a low risk of complications ${ }^{[5]}$, such as bleeding and vascular injury. Additionally, it requires special equipment and well-trained doctors. These limitations prohibit it from being a routine means of large-scale diagnosis for $\mathrm{PH}$. D-ECHO is another effective tool for early screening of $\mathrm{PH}$. It reveals the direction and speed of blood flow, cardiac chamber size, wall thickness and valve movement. The velocity of tricuspid regurgitation (VTR) is a common parameter that can be practically used to assess pulmonary artery pressure (PAP). Per recommendations, $\mathrm{PH}$ should be considered if VTR revealed by $\mathrm{D}-\mathrm{ECHO}$ is $\geq 2.9 \mathrm{~m} / \mathrm{s}$, which accounts for pulmonary artery systolic pressure $\geq 37 \mathrm{mmHg}^{[6]}$. However, the pulmonary artery pressure estimated by D-ECHO is often different from values measured by right heart catheterization, leading to misdiagnosis of $\mathrm{PH}^{[7]}$. Therefore, it is urgent to search for other efficient tools to assist in PH diagnosis. 
MicroRNAs (miRNAs) are a class of endogenous small non-coding RNAs at 19-25 nucleotides that can regulate gene expression post-transcriptionally ${ }^{[8,9]}$. Recent studies have shown that miRNAs can exist in the circulating blood as stable forms ${ }^{[10-12]}$, whose levels are closely related to diseases, indicating that plasma miRNAs can serve as biomarkers for disease $\operatorname{diagnosis}^{[13]}$. For example, we have found that miR-208a, a cardiac-specific miRNA, is significantly increased and has high sensitivity and specificity for the diagnosis of acute myocardial infarction $^{[14]}$. However, only scant evidence has shown that plasma miRNA levels are closely associated with $\mathrm{PH}$ pathological processes ${ }^{[15,16]}$. Therefore, in this study, we performed studies in experimental (monocrotaline-induced) and human pulmonary arterial hypertension to search for plasma miRNAs that can be used as a biomarker for $\mathrm{PH}$ diagnosis.

\section{Materials and methods}

\section{Establishment of a PH mouse model}

All animal experimental protocols complied with the guidelines of the Animal Care and Use Committee of the Second Military Medical University (Shanghai, China). The PH mouse model was established by single intraperitoneally injection of monocrotaline $(\mathrm{MCT})$ at $60 \mathrm{mg} / \mathrm{kg}$ body weight ${ }^{[17]}$. Thirtyeight $\mathrm{C} 57$ male mice were randomly divided into a control group $(n=10)$ and an MCT-induced PH group $(n=28)$. The PH group was then subdivided into $2 \mathrm{wk}-\mathrm{MCT}(n=11)$ and $4 \mathrm{wk}-$ MCT $(n=17)$ according to the time course of MCT injection.

\section{Histological analysis}

After anesthesia, sham or MCT-injected mice were sacrificed. The lungs and hearts were removed, fixed with $4 \%$ paraformaldehyde overnight and then embedded in paraffin. Paraffin sections $(4 \mu \mathrm{m})$ were stained with hematoxylin and eosin (H\&E).

\section{Plasma collection and storage}

Venous blood samples from mice or patients were collected before surgery and anti-coagulated with EDTA. Within $0.5 \mathrm{~h}$ of collection, the blood samples were processed by centrifugation at $1500 \times g$ for $10 \mathrm{~min}$, followed by $12000 \times g$ for $10 \mathrm{~min}$ to remove cell debris. The supernatant was transferred to RNase/DNase-free tubes and stored at $-80{ }^{\circ} \mathrm{C}$.

RNA preparation and quantitative reverse transcriptase polymerase chain reaction (qRT-PCR)

Total RNA from plasma was isolated by TRI Reagent BD (MRC, TR126) according to the instructions of the manufacturer with modifications. MiRNAs were reverse transcribed by M-MLV reverse transcriptase with special stem-loop primers for miRNAs. Real-time quantitative PCR was performed using SYBR Green Master Mix (TOYOBO Co). The amount of target miRNA in $\mathrm{PH}$ samples relative to that in control samples was analyzed and given by $2^{-\Delta \Delta \mathrm{Ct}}$.

\section{Patient population}

The protocol of this project was performed according to the principles of the Declaration of Helsinki and approved by the Medical Ethics Committee in Shanghai Changhai Hospital. Written informed consent was obtained from all participants before enrollment. We assessed hospitalized patients who underwent right heart catheterization in the Department of Cardiology, Shanghai Changhai Hospital, from June 2013 to September 2014. Individuals with suspected PH were clinically diagnosed with symptoms, chest X-ray, electrocardiograph (ECG), and other suspected diagnosis. PH was determined by right heart catheterization. Individuals with pulmonary artery pressure $\leq 20 \mathrm{mmHg}$ were categorized into the control group. Patients were excluded if they had malignancy, infections, coronary heart disease, diabetes and abnormal liver or renal function. One-hundred and seven patients were included to analyze miRNA expression, including $53 \mathrm{PH}$ cases and 54 controls. All experimental data reflecting the general clinical condition of subjects were collected, including name, age, sex, height, weight, D-ECHO, right heart catheterization data, brain natriuretic peptide, serum glucose and lipids, and liver and kidney function.

\section{Statistical analysis}

All statistical analysis was performed using SPSS18.0 software. Quantitative data were evaluated to determine whether they followed a normal distribution and were expressed as the mean \pm standard deviation. For the normal distribution data, the differences between two groups were compared using Student's $t$-test and among groups were analyzed using one-way ANOVA. Receiver operating characteristic curves (ROCs) were established for discriminating $\mathrm{PH}$ patients from the control group, and the area under the curve (AUC) was used to determine the diagnostic value of the biological indicators of PH. All $P$-values are two-sided, and a value less than 0.05 was considered a statistically significant difference.

\section{Results}

MiRNAs were dynamically altered in healthy and PH human plasma according to microarray analysis

To gain insight into the profile of miRNAs in the plasma of $\mathrm{PH}$ and non-PH individuals, microarray-based analysis was performed with total RNA extracted from two plasma pools collected from $4 \mathrm{PH}$ patients or 4 non-PH controls. Of all detected miRNAs, miR-451 was the top-ranked miRNA in the plasma of both groups. Based on 2-fold or greater changes, $189 \mathrm{miR}-$ NAs could be detected as being different between PH individuals and controls, including 162 decreased miRNAs and 37 increased miRNAs. These miRNAs were selected to show their relative enrichment in the plasma in Figure 1. The top 10 highest miRNAs in the controls were all decreased miRNAs, including miR-150-3p, miR-4327, miR-4534, miR-1229-5p, miR4530, miR-5100, miR-6124, mir-320d, miR-1273g-3p and miR126-3p. Among them, miR-150 was the most significantly decreased miRNA. The top 14 ranked miRNAs in the $\mathrm{PH}$ group were all increased miRNAs. As displayed in Figure 1B, miR-3162 was the most markedly increased miRNA in the plasma of the $\mathrm{PH}$ patient population. 
A

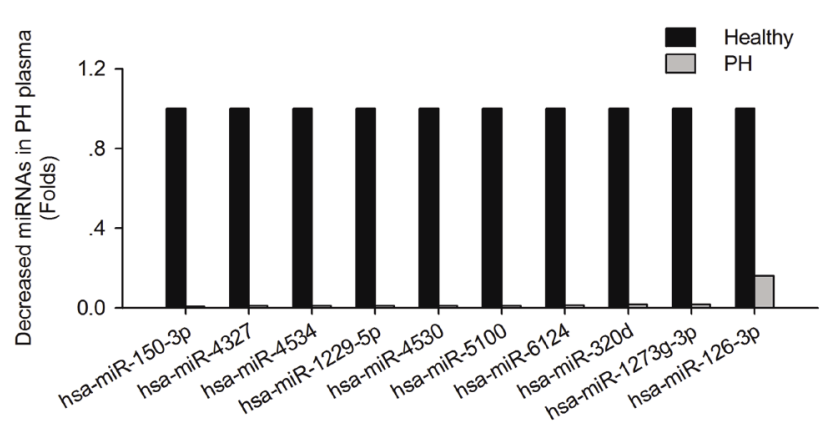

B

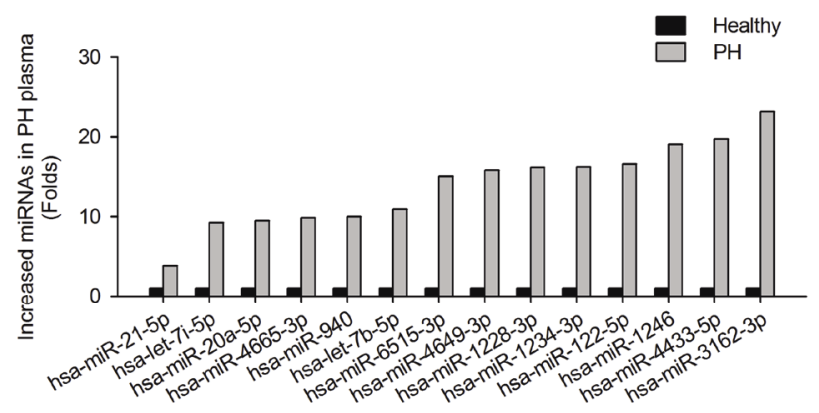

Figure 1. Altered miRNAs in plasma from healthy and $\mathrm{PH}$ patient populations using microarray analysis. A total of 189 miRNAs were detected as being different between healthy and $\mathrm{PH}$ patient populations by a 2-fold change, including 162 decreased miRNAs and 37 increased miRNAs. The top 10 decreased miRNAs (A) and top 14 increased miRNAs (B) in the PH patient population were revealed. MiR-150 was the most significantly decreased miRNA and miR-3162 the most markedly increased miRNA in the plasma of the $\mathrm{PH}$ patient population.

\section{Detection of miRNAs in the plasma of $\mathrm{PH}$ and control mice using} qRT-PCR

To test whether the miRNAs indicated above were dynamically regulated in the plasma of $\mathrm{PH}$ and control groups, a $\mathrm{PH}$ mouse model was established by single intraperitoneal injection of MCT at $60 \mathrm{mg} / \mathrm{kg}$ body weight. Animals subjected to saline were used as controls. After 2 weeks of MCT administration, the mice began to show tachypnea. The mice in the control group did not show any tachypnea. The mice were sacrificed 2 or 4 weeks after MCT injection (2wk-MCT or $4 \mathrm{wk}-$ $\mathrm{MCT}$ ). Two mice died in the 2 wk-MCT group, while 4 mice died in the $4 w k-M C T$ group. No mice in the control group died at these two time points. Using H\&E staining analysis, we observed that compared to controls, the pulmonary artery walls of lung tissues from $\mathrm{PH}$ mice were more thickened and infiltrated with more inflammatory cells (Figure 2A). The composition of the infiltrates may be inflammatory cells, thrombosis, and proliferative smooth muscle cells, similar to previous observations $\mathrm{s}^{[18-20]}$. Additionally, the right ventricles of hearts from $\mathrm{PH}$ mice displayed more hypertrophy than the control group (Figure 2B). Furthermore, the expression of cardiac hypertrophic markers was detected using qRT-PCR. As expected, the expression levels of $\mathrm{Nppa}$ and $m y \mathrm{~h} 7$ were significantly up-regulated, and myh 6 was markedly down-regulated in right ventricles from the $4 \mathrm{wk}-\mathrm{MCT}$-injected mice compared to the control (Figure 2C).

After establishment and verification of the $\mathrm{PH}$ mouse model, we selected a collection of miRNAs from our present and previous microarray, as well as microarrays from other groups ${ }^{[15]}$, for verification using qRT-PCR. These miRNAs are miR-21, miR-23b, miR-26a, miR-126, miR-145, miR-150, miR191, miR-204, miR-320, miR-451, miR-1246, and miR-3162. As reported, miR-16 was used as an endogenous control for data normalization. Those miRNAs whose $\mathrm{Ct}$ value was above 40 were defined as hard to detect with routine qRT-PCR, including miR-23b, miR-26a, miR-204, miR-320, miR-1246 and miR3162. Of the detectable candidate miRNAs, miR-126, miR-145, miR-191, miR-21 and miR-150 were found to have no difference between the control and PH mice (Figure 3). The relative qualities of individual mice are demonstrated in Figure 3. Interestingly, compared with the control, an early decreased level of plasma miR-451 can be observed in the 2 wk-MCT group, but no further decrease was detected in the 4wk-MCT group compared to the 2 wk-MCT group (Figure 3).

\section{MiR-451 was significantly decreased in the plasma of $\mathrm{PH}$ patients}

To determine the plasma level of miRNAs in PH patients, $5 \mathrm{~mL}$ blood was collected from individuals with suspected $\mathrm{PH}$ that underwent right heart catheterization. According to the RHC results, individuals were divided into two groups: the control group (mPAP $\leq 20 \mathrm{mmHg}$ ) and $\mathrm{PH}$ group (mPAP $\geq 25 \mathrm{mmHg}$ ). Clinical data of these enrolled patients were collected. In this case-control experiment, one-hundred and seven individuals were included to analyze miRNA expression, including $53 \mathrm{PH}$ cases and 54 controls. The relative clinical data reflecting the general clinical condition were collected and are shown in Table 1. Several statistical indexes were not different between the control and $\mathrm{PH}$ patients, including age, sex, height, weight, SBP, DBP, MBP, HR, LVEF, FBG, sCR, WBC, NEAU and sUA. NT-proBNP, SPAP, DPAP and mPAP were significantly up-regulated, consistent with previous reports. Then, the 12 candidate miRNAs indicated above were detected using qRT-PCR. In the RNA samples of the control and patient group, the plasma levels of miR-145, miR-191, miR-26a, miR-23b, miR-204, miR-3162, and miR-1246 were either not detected or displayed CT values that were too large. Additionally, there was no difference in the circulating levels of miR-126, miR-21, miR-320 and miR-150 between the PH and control groups (Figure 4). Interestingly, consistent with the animal results, the plasma level of miR-451 was markedly decreased in the $\mathrm{PH}$ patient population compared to the control group, indicating that circulating miR-451 might be able to serve as a biomarker for PH (Figure 4).

\section{Comparison of the diagnostic value of circulating miR-451 with D-ECHO for PH using the ROC}

To assess the clinical diagnostic value of circulating miR451 as a biomarker of $\mathrm{PH}, \mathrm{ROC}$ analysis was performed on data from 107 individuals, including $54 \mathrm{PH}$ and 53 controls. The ROC of miR- 451 with AUC $=0.710$ reflected a moderate 
A
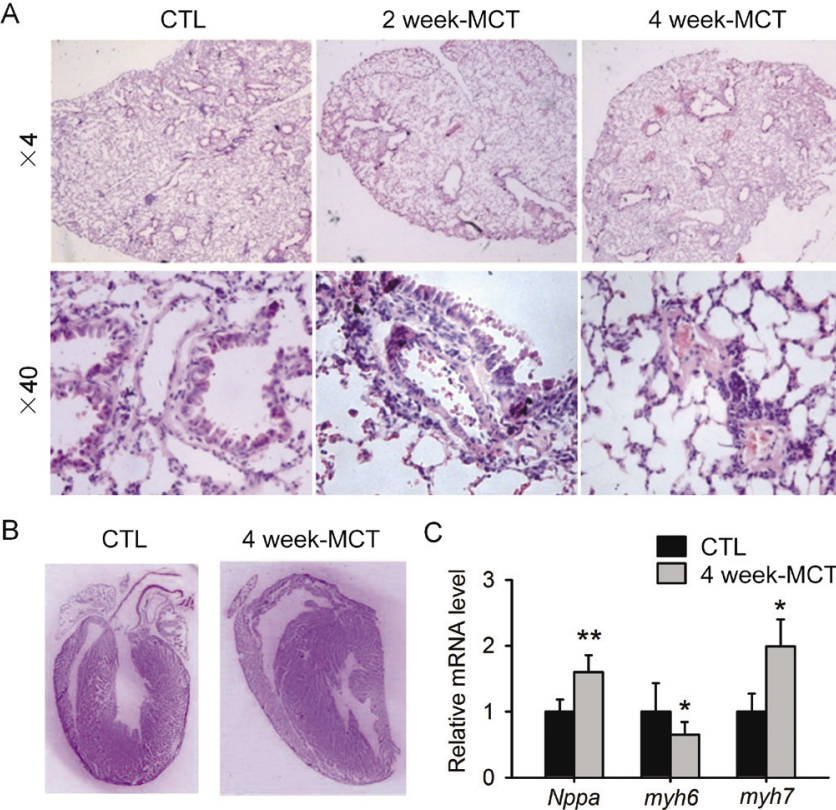

Figure 2. Establishment of $\mathrm{PH}$ mouse model. A PH mouse model was established by single intraperitoneal injection of MCT at $60 \mathrm{mg} / \mathrm{kg}$ body weight. Animals subjected to saline were used as controls. Mice were sacrificed at 2 or 4 weeks after administration. Compared to controls, lung tissue from the $\mathrm{PH}$ model group was detected as having a more thickened pulmonary artery wall, more inflammatory cell infiltration and more micro-thrombosis at both 2 and 4 weeks after MCT administration (A). Myocardial hypertrophy of the right heart ventricle was also observed in the PH model group (B). Hypertrophic markers were detected by qRT-PCR. Expression of $\beta$-myosin heavy chain (myh7) and atria natriuretic peptide (Nppa) was significantly up-regulated, while expression of $\alpha$-myosin heavy chain ( $\alpha$-MHC, myh6) was markedly down-regulated in the right heart ventricle from MCT-administered mice compared to control mice (C). ${ }^{*} P<0.05,{ }^{* *} P<0.01$.

diagnostic value between $\mathrm{PH}$ and control groups, indicating that circulating miR-451 could be used as a diagnostic reference for PH (Figure 5A). These values are consistent with the current clinical status of noninvasive diagnosis for PH. As $\mathrm{D}-\mathrm{ECHO}$ is a commonly used diagnostic indicator for $\mathrm{PH}$, we therefore analyzed the clinical diagnostic value of $\mathrm{D}-\mathrm{ECHO}$ in these individuals. The AUC of the ROC for D-ECHO is 0.766 for $\mathrm{PH}$ diagnosis, indicating that $\mathrm{D}-\mathrm{ECHO}$ is similar to miR451 , with a moderate diagnostic value (Figure 5B). Furthermore, we assessed the value of combining circulating miR-451 with $\mathrm{D}-\mathrm{ECHO}$ for $\mathrm{PH}$ diagnosis using a stepwise regression method. The combination of miR-451 with D-ECHO, with AUC $=0.825$ (Figure 5C), had a better diagnostic value than either miR-451 or D-ECHO alone.

\section{Discussion}

In the present study, we showed that circulating miR-451 was significantly decreased in PH model mice vs control mice and in patients suffering from $\mathrm{PH}$ vs control patients, indicating that plasma miR-451 has a diagnostic value for $\mathrm{PH}$ and is a potential biomarker of $\mathrm{PH}$. Our results provide important insights into PH diagnosis.

Currently, right heart catheterization is the best method and gold standard for $\mathrm{PH}$ diagnosis. The inclusion criteria for patients with $\mathrm{PH}$ were based on pulmonary artery pressure $\geq 25 \mathrm{mmHg}$, while for controls, the pulmonary artery pressure was $\leq 20 \mathrm{mmHg}$. Individuals who had a pulmonary artery pressure between 20 and $25 \mathrm{mmHg}$ were not enrolled in the present study. D-ECHO is another commonly used diagnostic indicator for early screening of $\mathrm{PH}$. As recommended ${ }^{[6]}$, here, we grouped the individuals with VTR $\geq 2.9 \mathrm{~m} / \mathrm{s}$, accounting for pulmonary artery systolic pressure $\geq 37 \mathrm{mmHg}$, into the $\mathrm{PH}$ group, while those with VTR $<2.9 \mathrm{~m} / \mathrm{s}$ were placed in the control group. Compared with right heart catheterization, the clinical diagnostic value of D-ECHO in these patients, indicated by the AUC of the ROC, is 0.766 . As commonly recognized, an AUC value of 0.7-0.9 indicates a moderate diagnostic value, and $>0.9$ is high. The AUC of the ROC is 0.766 , indicating that $\mathrm{D}-\mathrm{ECHO}$ could serve as a moderate marker for $\mathrm{PH}$. These results was consistent with previous reports indicating that approximately $60 \%$ of the ultrasound estimation of pulmonary artery pressure was at least $10 \mathrm{mmHg}$ lower than that measured by right heart catheterization ${ }^{[7]}$. There are many missed examinations and a significant misdiagnosis rate for $\mathrm{D}-\mathrm{ECHO}^{[21,22]}$. In the present study, we also assessed the clinical diagnostic value of circulating miR-451 as a biomarker of PH. The AUC of the ROC is 0.710 for circulating miR-451 in PH diagnosis, which is slightly less than the value for $\mathrm{D}-\mathrm{ECHO}$, but also reflects a moderate diagnostic value. In the current clinical status of $\mathrm{PH}$, there are no gold-standard non-invasive diagnostic methods for $\mathrm{PH}$. For this reason, we supposed that both D-ECHO and miR-451 could serve as diagnostic references for $\mathrm{PH}$. As expected, the combination of circulating miR-451 with D-ECHO for PH diagnosis was superior, with $\mathrm{AUC}=0.825$, to either miR-451 or D-ECHO.

In previous studies, three categories of $\mathrm{PH}$ biomarkers have been proposed and explored. The first category is biomarkers from hearts, such as cTnT, BNP and NT-proBNP ${ }^{[23-26]}$. However, cTnT can only be detected at the late stage of $\mathrm{PH}$ when the heart is severely injured. BNP and NT-proBNP have been shown to be independent predictors for $\mathrm{PH}$ prognosis. A normal level of BNP or NT-proBNP may help to rule out PH, but due to their fast degradation, neither can serve as biomarkers. The second category is inflammation-related biomarkers, such as CRP and IL-6, whose specificity for biomarkers of PH diagnosis is not sufficient ${ }^{[27,28]}$. It remains to be investigated whether the combination of CRP or IL6 with D-ECHO or miR451 could serve as a diagnostic reference for $\mathrm{PH}$. The third category is metabolism-related molecules, such as $5-\mathrm{HT}^{[29,30]}$, whose potential as a biomarker for $\mathrm{PH}$ suffers from a lack of evidence. To date, there is still a lack of efficient and specific biomarkers for $\mathrm{PH}$. In this study, we showed that circulating miR-451 could be used as a moderate reference for PH. Further testing is required to determine whether miR-451 can serve as a serum biomarker to evaluate the progress of $\mathrm{PH}$. Good biomarkers should ideally fulfill the following criteria: high sensitivity, high specificity and sufficiently closely corre- 
Table1. General characteristics of the two groups of patients.

\begin{tabular}{lll}
\hline & Control group $(n=54)$ & PAH group $(n=53)$ \\
\hline Age, year & $33.83 \pm 15.24$ & $46.46 \pm 15.33$ \\
Male, $n$ (\%) & $22(40.74 \%)$ & $19(35.85 \%)$ \\
Height, cm & $161.87 \pm 12.56$ & $162.24 \pm 8.88$ \\
Weight, kg & $56.45 \pm 13.95$ & $60.60 \pm 16.52$ \\
SBP, mmHg & $115.83 \pm 10.68$ & $120.92 \pm 15.46$ \\
DBP, mmHg & $73.20 \pm 8.73$ & $76.55 \pm 10.22$ \\
MBP, mmHg & $88.85 \pm 7.94$ & $90.04 \pm 12.27$ \\
HR, bpm & $75.57 \pm 8.19$ & $72.78 \pm 9.50$ \\
SPAP, mmHg & $29.23 \pm 5.40$ & $53.27 \pm 18.36^{* *}$ \\
DPAP, mmHg & $9.92 \pm 3.09$ & $24.43 \pm 13.93^{* *}$ \\
mPAP, mmHg & $16.81 \pm 2.57$ & $35.12 \pm 14.24^{* *}$ \\
LVEF, \% & $65.58 \pm 4.11$ & $63.38 \pm 5.87$ \\
FBG, mmol/L & $5.10 \pm 0.95$ & $5.22 \pm 0.87$ \\
sCR, mmol/L & $63.72 \pm 15.30$ & $63.90 \pm 12.30$ \\
WBC, $x 10^{9} / \mathrm{L}$ & $5.86 \pm 1.42$ & $6.13 \pm 2.07$ \\
NEAU, \% & $57.53 \pm 9.58$ & $66.57 \pm 10.65$ \\
ALT, U/L & $16.24 \pm 11.99$ & $20.72 \pm 10.68$ \\
sUA, mmol/L & $0.31 \pm 0.09$ & $0.36 \pm 0.11$ \\
NT-proBNP, $\mathrm{pg} / \mathrm{mL}$ & $56(5 \sim 293)$ & $194(5 \sim 5252)^{* *}$ \\
\hline
\end{tabular}

${ }^{* *} P<0.01,{ }^{\star}$ Mann-Whitney test.

lated to or able to reflect the progress of disease. Regrettably, none of the biomarkers for PH can totally meet these 3 criteria. Due to the limited number of patients enrolled in the present study, we cannot conclude that miR-451 is a serum biomarker able to evaluate the progress of $\mathrm{PH}$. Therefore, it remains to be investigated whether miR-451 can meet these 3 criteria in a large population.

Three strategies were used to choose candidate plasma miRNAs for qRT-PCR analysis in the animal model and largescale PH patient studies. First, a microarray was used to screen miRNAs with different levels in the plasma between the control and $\mathrm{PH}$ patient populations, among which a collection of miRNAs was further selected for verification in animal models or large $\mathrm{PH}$ patient populations, such as miR21, miR-150, miR-320d, miR-1246 and miR-3162. Several of these candidate miRNAs could not be detected, such as miR204, miR-1246 and miR-3162. Although miR-21 and miR-150 could be detected in the plasma of animal models, these two miRNAs were not observed to differ between control and $\mathrm{PH}$ patients, possibly due to different detection sensitivities and false positive rates between the microarray and qRT-PCR. In our opinion, based on previous reports, we think that qRTPCR is more reliable. It has been reported that miR-21 was increased in the plasma of $\mathrm{PH}$ patients ${ }^{[15]}$. However, in our qRT-PCR results, no up-regulation was detected, possibly due to different patient populations and different examination methods. Another principle was used to select candidate miRNAs. Tissue-specific expressed miRNAs may serve as a biomarker for $\mathrm{PH}$ with high specificity, such as miR-126 and miR-145. MiR-126, an endothelial cell-specific miRNA ${ }^{[31]}$, might be released into the blood upon endothelial cell injury. However, unexpectedly, miR-126 was decreased in our micro-
$\operatorname{miR}-451$

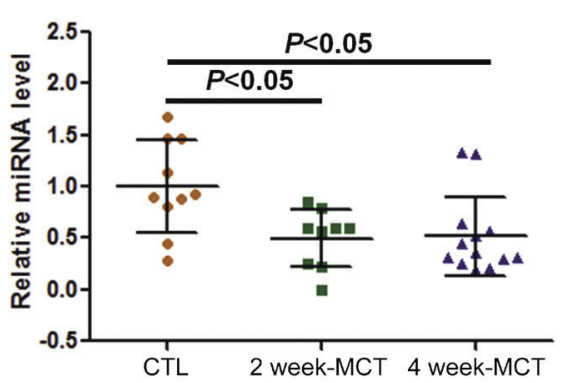

miR-145

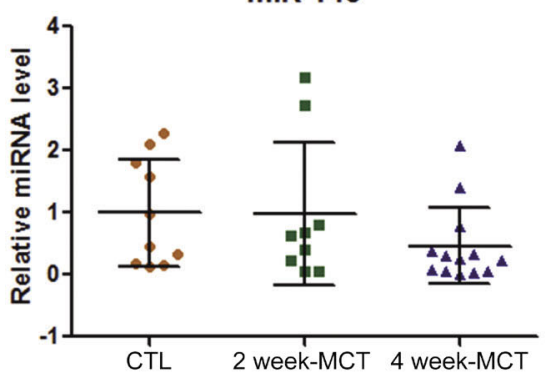

miR-21

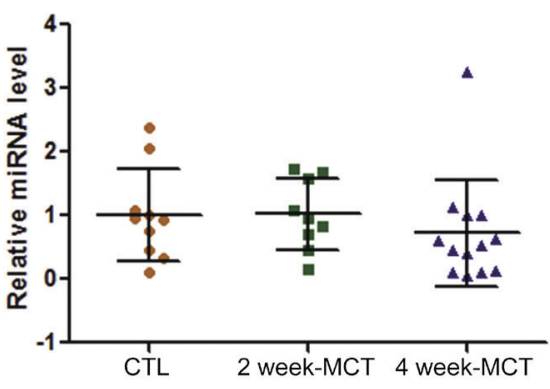

$\operatorname{miR}-150$

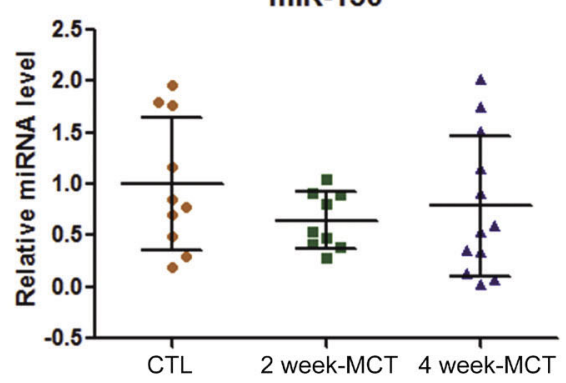

miR-126

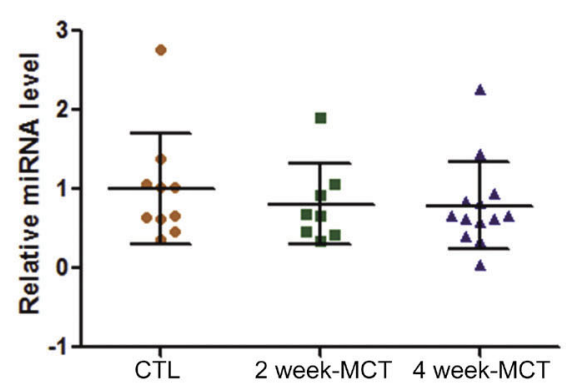

miR-191

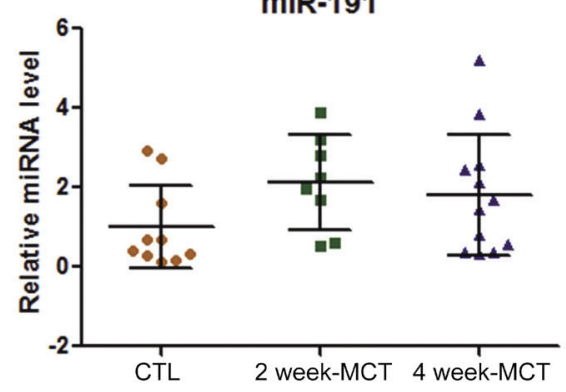

Figure 3. Analysis of candidate miRNAs in the plasma of WT and PH mice using qRT-PCR. Two miRNAs closely related to PH, i.e., miR-145 and miR-191, 4 miRNAs from the microarray results and 1 miRNA most enriched in the plasma were selected for detection by qRT-PCR. MiR-320d was not detected in both WT and PH mice. The plasma levels of miR-126, miR-145, miR-191, miR-21 and miR-150 were not significantly altered, while that of miR-451 was significantly lower in the 2 wk-MCT group and 4 wk-MCT group compared to the control group. The miR- 451 level was not different between the 2 wk-MCT and 4 wk-MCT groups. 


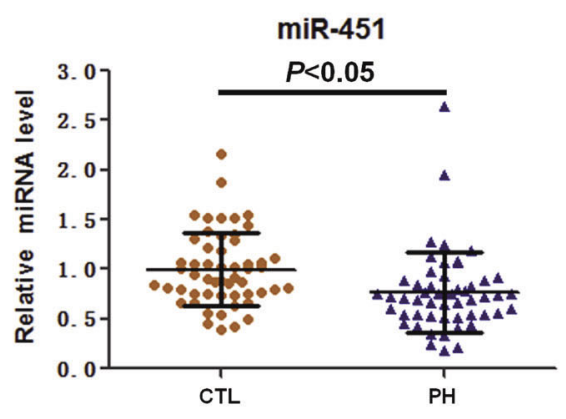

miR-21

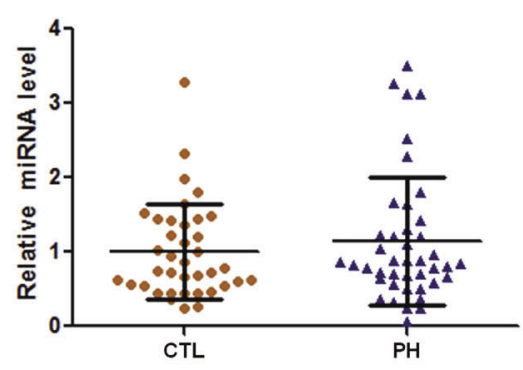

miR-320d
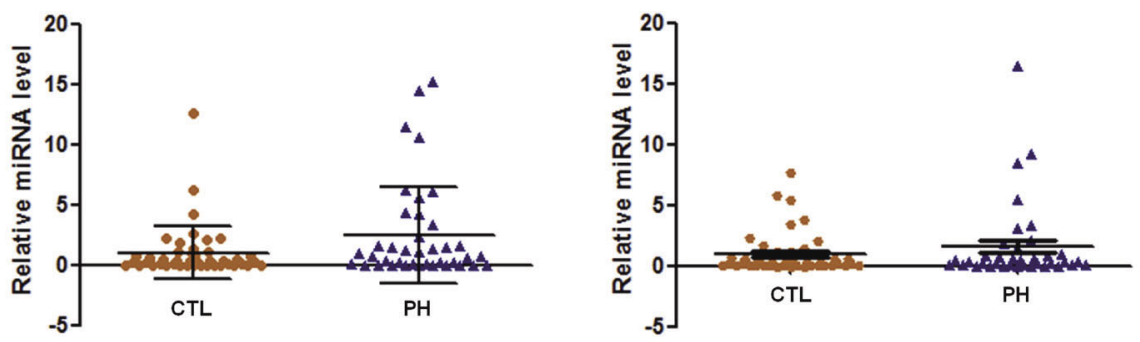

Figure 4. Assessment of the diagnostic value of circulating miR-451 with $\mathrm{D}-\mathrm{ECHO}$ for $\mathrm{PH}$. According to RHC results, patients were divided into a control group (mPAP $\leq 20 \mathrm{mmHg}$ ) and a PH group (mPAP $\geq 25 \mathrm{mmHg}$ ). In samples of serum from patients and controls, miR-145 and miR-191 were either not detected or displayed CT values that were too large. The plasma levels of miR-126, miR-21 miR-150, miR-451 and miR-320d were verified using qRTPCR. The levels of miR-126, miR-21 and miR-150 were not significantly different between PH patients and the control group. However, the level of miR451 decreased in the $\mathrm{PH}$ group compared to the control population.

array results and did not differ between the controls and $\mathrm{PH}$ patient population or the control and experimental animal models. Another miRNA, miR-145, was reported to be specifically expressed in smooth muscle cells and involved in vascular endothelium injury ${ }^{[32]}$. Unfortunately, no alteration of it was detected in the plasma of animal models by qRT-
PCR or in PH patients by microarray. Lastly, we examined plasma-enriched miRNAs as objects of investigation, among which miR-451 was the most enriched miRNA in both control and PH patient populations. Unexpectedly, in our animal model system, we found that miR-451 was significantly decreased in both the $2 \mathrm{wk}-\mathrm{MCT}$ and $4 \mathrm{wk}-\mathrm{MCT}$ groups after

\section{$\operatorname{miR}-451$}

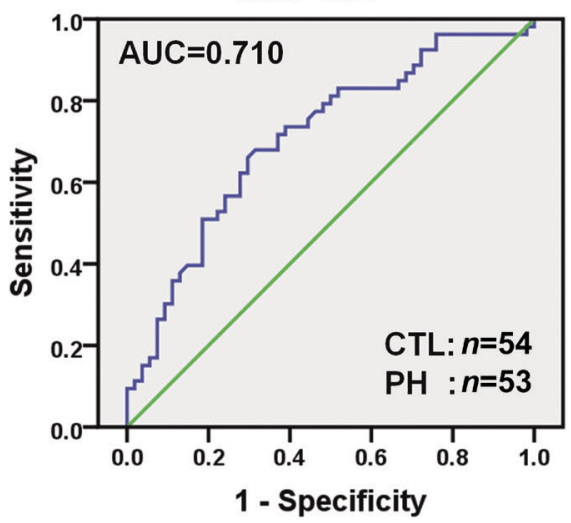

UCG

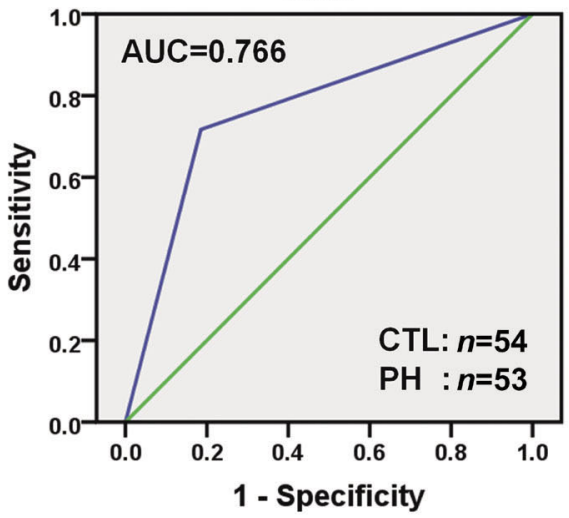

UCG+miR-451

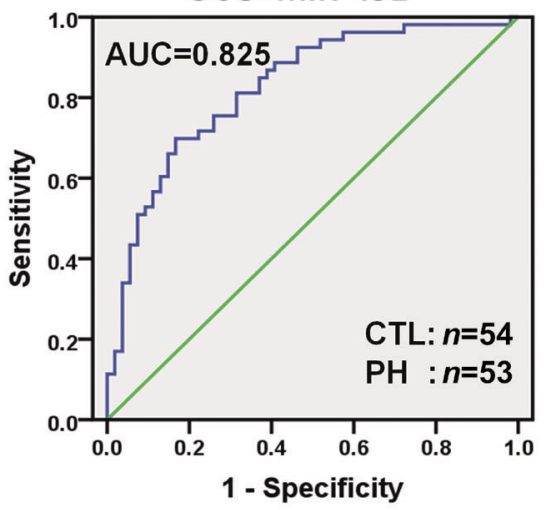

Figure 5. Compared to the normal control group, the relative blood level of miR-451 in PH diagnosis had a moderate diagnostic value. The area under the ROC (AUC) is 0.710 , with a standard error of 0.076 for the diagnosis of $\mathrm{PH}(P=0.004)$. The area of the $95 \%$ confidence interval $(0.625$ to 0.922$)$ does not include 0.5 , indicating that the diagnostic value of circulating miR-451 has significance $(A)$. It was comparable with $D-E C H O$ (AUC $=0.766$ ), which is used in clinics for PH diagnosis (B). The values of the combination of circulating miR-451 and D-ECHO for PH diagnosis were determined using a stepwise regression method. The combination of miR-451 with D-ECHO had a better diagnostic value than either miR-451 or D-ECHO alone, with $A \cup C=0.825(C)$. 
MCT administration, suggesting that miR-451 might decline in early stage modeling, but with no further decrease later. Therefore, down-regulation of miR-451 in both the 2wk-MCT and $4 \mathrm{wk}-\mathrm{MCT}$ groups after MCT administration might have some prognostic value for $\mathrm{PH}$. More interestingly, miR-451 was also significantly decreased in the plasma of $\mathrm{PH}$ patient populations. This was highly consistent between the studies of animal models and $\mathrm{PH}$ patient populations. The difference in the miR-451 plasma level between our qRT-PCR results and microarray results might be due to its enriched level, which was several folds higher than that of other miRNAs in plasma, possibly saturating the detection signals of the microarray to prohibit determination of the difference between the control and $\mathrm{PH}$ patient populations. However, using qRT-PCR, we observed that plasma miR-451 is different in animals and patient populations with normal and high pulmonary artery pressure. Future studies should determine when miR-451 begins to decrease during $\mathrm{PH}$ progress to determine whether miR-451 could be used as biomarker even before pulmonary artery remodeling.

In a comparison of our results with other reports, we noted that there were only a few studies showing that the levels of plasma miRNAs were closely associated with the pathological processes of PH. A survey of miRNA analysis using microarrays showed that miR-1246 decreased, while miR23, miR-130, and miR-191 increased in the plasma of $\mathrm{PH}$ patients ${ }^{[15]}$. Here, consistent with these results, we also found that miR-1246 declined, while miR-23 increased in our microarray. However, in contrast with these results, miR-130 and miR-191 were markedly decreased, possibly due to the different backgrounds of patients and controls. To further evaluate the results for miR-191, we determined its level in the PH model using qRT-PCR, showing that miR-191 was not altered in the animal model. It remains to be investigated whether miR-191 could potentially serve as a biomarker for $\mathrm{PH}$. Another small-sized patient population analysis showed that plasma miR-204 significantly declined in $\mathrm{PH}^{[16]}$. In contrast, we did not observe any alteration of miR-204 in the plasma of $\mathrm{PH}$ individuals in our microarray results. Thus, we did not choose miR-204 as a potential target to validate its potential as a biomarker for $\mathrm{PH}$ in animal models and the patient population study using qRT-PCR.

It has been revealed that several miRNAs are involved in the pathogenesis of $\mathrm{PH}, \mathrm{miR}-150^{[33,34]}, \operatorname{miR}-21^{[35,36]}, \operatorname{miR}-126^{[37]}$, miR- $145^{[38]}$ and miR-424 $4^{[39]}$. These miRNAs might have implications in the pathophysiology or therapeutics of PH. For example, miR-424 has been shown to correlate with markers of $\mathrm{PH}$ disease severity and can be taken up by cardiomyocytes to sustain the bone morphogenetic protein receptor 2 signaling pathway ${ }^{[39]}$. As miR-451 is the most enriched miRNA in the plasma, where and how miR-451 arise and whether miR451 is just an indicator for $\mathrm{PH}$ or is involved in regulating $\mathrm{PH}$ progress are all topics that warrant further investigation. In our PH mouse model, we observed that the pulmonary artery walls of PH mice were infiltrated with cellular compositions. Identifying the cell profile and gene expression in the lesions of the MCT model might provide information about where miR-451 was released and whether serum miR-451 functions in $\mathrm{PH}$ progress. It was previously reported that the composition of these infiltrates induced by MCT was thrombosis, inflammatory cells, and proliferative smooth muscle cells ${ }^{[18-20]}$. miRNAs have been reported to exist as microparticles, apoptosis bodies and other forms and can shuttle between different cell types ${ }^{[10-12,40]}$. We posit that a decreased level of serum miR-451 might affect the functions of endothelial cells or smooth muscle cells. The endothelial cells or smooth muscle cells might absorb serum miR-451 to fulfill their physiological or pathological functions, such as apoptosis and proliferation. It has been reported that transient, but not genetic, loss of miR-451 is protective in the development of $\mathrm{PH}^{[41]}$. Therefore, more research is warranted to validate whether circulating miR-451 promotes infiltrates in the pulmonary artery walls during $\mathrm{PH}$ processes and to determine how serum miR-451 interferes with the degradation or translational repression of target mRNA in endothelial or smooth muscle cells.

Taken together, circulating miR-451 might be able to serve as a biomarker for $\mathrm{PH}$. It has moderate diagnostic value comparable to D-ECHO. The combination of miR-451 with D-ECHO might have implications for $\mathrm{PH}$ therapeutic outcomes.

\section{Acknowledgements}

This work was supported in part by the National Natural Science Foundation of China (91739301, 81301855 and 91339205); the National Key Research \& Development Program of China (2017YFA0103700) and the Strategic Priority Research Program of the Chinese Academy of Science (XDA16020903).

\section{Author contribution}

Xiao-wei SONG and Qing JING designed the project; Xiao-wei SONG, Lu-lu ZOU and Ling CUI performed the experiments; Song-hua LI, Yong-wen QIN and Xian-xian ZHAO collected the plasma and interpreted clinical data; and Xiao-wei SONG and Qing JING wrote the manuscript.

\section{Abbreviations}

$\mathrm{PH}$, pulmonary hypertension; MCT, monocrotaline; ROC, receiver operating characteristic curve; D-ECHO, Doppler echocardiography; AUC, area under the curve; mPAP, mean pulmonary artery pressure; $\mathrm{RHC}$, right heart catheterization; VTR, velocity of tricuspid regurgitation; PAP, pulmonary artery pressure.

\section{References}

1 Archer S, Rich S. Primary pulmonary hypertension: a vascular biology and translational research "Work in progress". Circulation 2000; 102: 2781-91.

2 Humbert M, Sitbon O, Chaouat A, Bertocchi M, Habib G, Gressin V, et al. Survival in patients with idiopathic, familial, and anorexigenassociated pulmonary arterial hypertension in the modern management era. Circulation 2010; 122: 156-63.

3 Humbert M, Sitbon O, Chaouat A, Bertocchi M, Habib G, Gressin V, et al. Pulmonary arterial hypertension in France: results from a national registry. Am J Respir Crit Care Med 2006; 173: 1023-30. 
4 McLaughlin VV, Archer SL, Badesch DB, Barst RJ, Farber HW, Lindner JR, et al. ACCF/AHA 2009 expert consensus document on pulmonary hypertension: a report of the American College of Cardiology Foundation Task Force on Expert Consensus Documents and the American Heart Association: developed in collaboration with the American College of Chest Physicians, American Thoracic Society, Inc, and the Pulmonary Hypertension Association. Circulation 2009; 119: 2250-94.

5 Hoeper MM, Lee SH, Voswinckel R, Palazzini M, Jais X, Marinelli A, et al. Complications of right heart catheterization procedures in patients with pulmonary hypertension in experienced centers. J Am Coll Cardiol 2006; 48: 2546-52.

6 Hoeper MM, Ghofrani HA, Gorenflo M, Grunig E, Schranz D, Rosenkranz S. Diagnosis and treatment of pulmonary hypertension: European guidelines 2009. Pneumologie 2010; 64: 401-14.

7 Kiatchoosakun S, Wongvipaporn C, Nanagara R, Hoit BD. Right ventricular systolic pressure assessed by echocardiography: a predictive factor of mortality in patients with scleroderma. Clin Cardiol 2011; 34: 488-93.

8 Bartel DP. MicroRNAs: genomics, biogenesis, mechanism, and function. Cell 2004; 116: 281-97.

9 Li Q, Song XW, Zou J, Wang GK, Kremneva E, Li XQ, et al. Attenuation of microRNA-1 derepresses the cytoskeleton regulatory protein twinfilin-1 to provoke cardiac hypertrophy. J Cell Sci 2010; 123: 2444-52.

10 Akao Y, lio A, Itoh T, Noguchi S, Itoh Y, Ohtsuki Y, et al. Microvesiclemediated RNA molecule delivery system using monocytes/ macrophages. Mol Ther 2011; 19: 395-9.

11 Hunter MP, Ismail N, Zhang X, Aguda BD, Lee EJ, Yu L, et al. Detection of microRNA expression in human peripheral blood microvesicles. PLoS One 2008; 3: e3694.

12 Zernecke A, Bidzhekov K, Noels H, Shagdarsuren E, Gan L, Denecke B, et al. Delivery of microRNA-126 by apoptotic bodies induces CXCL12dependent vascular protection. Sci Signal 2009; 2: ra81.

13 Tanaka M, Oikawa K, Takanashi M, Kudo M, Ohyashiki J, Ohyashiki K, et al. Down-regulation of miR-92 in human plasma is a novel marker for acute leukemia patients. PLoS One 2009; 4: e5532.

14 Wang GK, Zhu JQ, Zhang JT, Li Q, Li Y, He J, et al. Circulating microRNA: a novel potential biomarker for early diagnosis of acute myocardial infarction in humans. Eur Heart J 2010; 31: 659-66.

15 Wei C, Henderson H, Spradley C, Li L, Kim IK, Kumar S, et al. Circulating miRNAs as potential marker for pulmonary hypertension. PLoS One 2013; 8: e64396.

16 Courboulin A, Paulin R, Giguere NJ, Saksouk N, Perreault T, Meloche J, et al. Role for miR-204 in human pulmonary arterial hypertension. J Exp Med 2011; 208: 535-48.

17 Kay JM, Harris P, Heath D. Pulmonary hypertension produced in rats by ingestion of Crotalaria spectabilis seeds. Thorax 1967; 22: 176-9.

18 Nogueira-Ferreira R, Vitorino R, Ferreira R, Henriques-Coelho T. Exploring the monocrotaline animal model for the study of pulmonary arterial hypertension: a network approach. Pulm Pharmacol Ther 2015; 35: 8-16.

19 Ryan JJ, Marsboom G, Archer SL. Rodent models of group 1 pulmonary hypertension. Handb Exp Pharmacol 2013; 218: 105-49.

20 Sehgal PB, Mukhopadhyay S. Dysfunctional intracellular trafficking in the pathobiology of pulmonary arterial hypertension. Am J Respir Cell Mol Biol 2007; 37: 31-7.

21 Arcasoy SM, Christie JD, Ferrari VA, Sutton MS, Zisman DA, Blumenthal NP, et al. Echocardiographic assessment of pulmonary hypertension in patients with advanced lung disease. Am J Respir Crit Care Med 2003; 167: 735-40.
22 Fisher MR, Criner GJ, Fishman AP, Hassoun PM, Minai OA, Scharf SM, et al. Estimating pulmonary artery pressures by echocardiography in patients with emphysema. Eur Respir J 2007; 30: 914-21.

23 Leuchte HH, Holzapfel M, Baumgartner RA, Neurohr C, Vogeser M, Behr J. Characterization of brain natriuretic peptide in long-term followup of pulmonary arterial hypertension. Chest 2005; 128: 2368-74.

24 Giannakoulas G, Dimopoulos K, Bolger AP, Tay EL, Inuzuka R, Bedard $\mathrm{E}$, et al. Usefulness of natriuretic Peptide levels to predict mortality in adults with congenital heart disease. Am J Cardiol 2010; 105: 869-73.

25 Fijalkowska A, Kurzyna M, Torbicki A, Szewczyk G, Florczyk M, Pruszczyk $\mathrm{P}$, et al. Serum $\mathrm{N}$-terminal brain natriuretic peptide as a prognostic parameter in patients with pulmonary hypertension. Chest 2006; 129: 1313-21.

26 Nagaya N, Nishikimi T, Uematsu M, Satoh T, Kyotani S, Sakamaki F, et al. Plasma brain natriuretic peptide as a prognostic indicator in patients with primary pulmonary hypertension. Circulation 2000; 102: 865-70.

27 Sztrymf B, Souza R, Bertoletti L, Jais X, Sitbon O, Price LC, et al. Prognostic factors of acute heart failure in patients with pulmonary arterial hypertension. Eur Respir J 2010; 35: 1286-93.

28 Quarck R, Nawrot T, Meyns B, Delcroix M. C-reactive protein: a new predictor of adverse outcome in pulmonary arterial hypertension. J Am Coll Cardiol 2009; 53: 1211-8.

29 Herve P, Launay JM, Scrobohaci ML, Brenot F, Simonneau G, Petitpretz $\mathrm{P}$, et al. Increased plasma serotonin in primary pulmonary hypertension. Am J Med 1995; 99: 249-54.

30 MacLean MR, Herve P, Eddahibi S, Adnot S. 5-Hydroxytryptamine and the pulmonary circulation: receptors, transporters and relevance to pulmonary arterial hypertension. Br J Pharmacol 2000; 131: 161-8.

31 Wang S, Aurora AB, Johnson BA, Qi X, McAnally J, Hill JA, et al. The endothelial-specific microRNA miR-126 governs vascular integrity and angiogenesis. Dev Cell 2008; 15: 261-71.

32 Cheng Y, Liu X, Yang J, Lin Y, Xu DZ, Lu Q, et al. MicroRNA-145, a novel smooth muscle cell phenotypic marker and modulator, controls vascular neointimal lesion formation. Circ Res 2009; 105: 158-66.

33 Chen M, Shen C, Zhang Y, Shu H. MicroRNA-150 attenuates hypoxiainduced excessive proliferation and migration of pulmonary arterial smooth muscle cells through reducing HIF-1alpha expression. Biomed Pharmacother 2017; 93: 861-8.

34 Rhodes CJ, Wharton J, Boon RA, Roexe T, Tsang H, Wojciak-Stothard $B$, et al. Reduced microRNA-150 is associated with poor survival in pulmonary arterial hypertension. Am J Respir Crit Care Med 2013; 187: 294-302.

35 Zhu B, Gong Y, Yan G, Wang D, Qiao Y, Wang Q, et al. Down-regulation of IncRNA MEG3 promotes hypoxia-induced human pulmonary artery smooth muscle cell proliferation and migration via repressing PTEN by sponging miR-21. Biochem Biophys Res Commun 2018; 495: 2125-32.

36 Yang S, Banerjee S, Freitas A, Cui H, Xie N, Abraham E, et al. miR-21 regulates chronic hypoxia-induced pulmonary vascular remodeling. Am J Physiol Lung Cell Mol Physiol 2012; 302: L521-9.

37 Potus F, Ruffenach G, Dahou A, Thebault C, Breuils-Bonnet S, Tremblay E, et al. Downregulation of microRNA-126 contributes to the failing right ventricle in pulmonary arterial hypertension. Circulation 2015; 132: 932-43.

38 Caruso P, Dempsie Y, Stevens HC, McDonald RA, Long L, Lu R, et al. A role for miR-145 in pulmonary arterial hypertension: evidence from mouse models and patient samples. Circ Res 2012; 111: 290-300.

39 Baptista R, Marques C, Catarino S, Enguita FJ, Costa MC, Matafome $\mathrm{P}$, et al. MicroRNA-424(322) as a new marker of disease progression in pulmonary arterial hypertension and its role in right ventricular 
hypertrophy by targeting SMURF1. Cardiovasc Res 2018; 114: 53-64.

40 Chen X, Liang H, Zhang J, Zen K, Zhang CY. Secreted microRNAs: a new form of intercellular communication. Trends Cell Biol 2012; 22: 125-32.

41 Grant JS, Morecroft I, Dempsie Y, van Rooij E, MacLean MR, Baker $\mathrm{AH}$. Transient but not genetic loss of miR-451 is protective in the development of pulmonary arterial hypertension. Pulm Circ 2013; 3: 840-50.
This work is licensed under the Creative

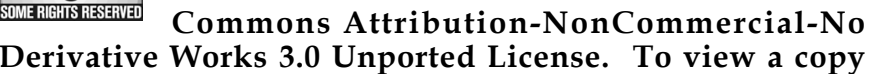
of this license, visit http://creativecommons.org/licenses/ by-nc-nd/3.0/

(C) The Author(s) 2018 